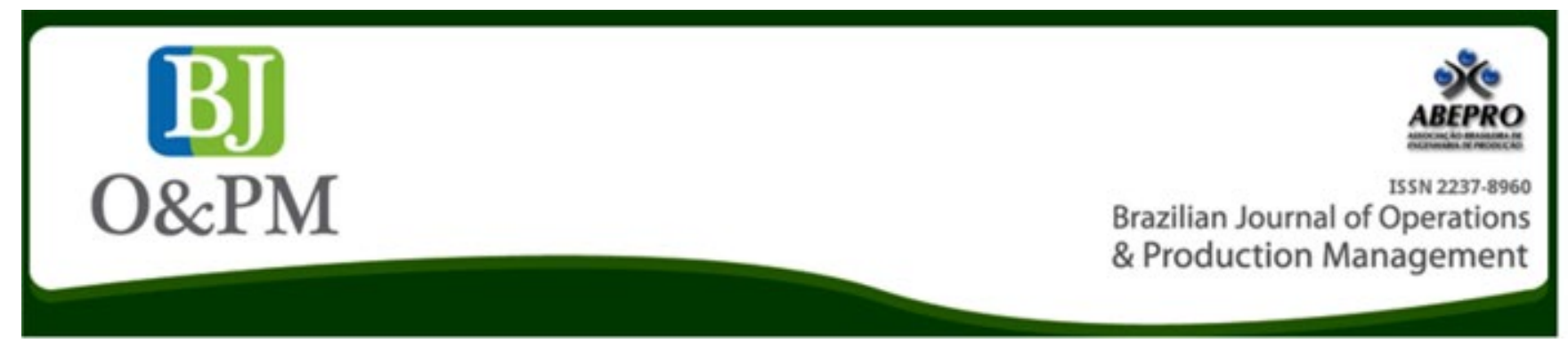

\title{
WHAT IS THE IDEAL SCENARIO FOR CIRCULAR ECONOMY TO OCCUR? A CASE STUDY OF THE CIRCE PROJECT
}

\section{Hanna Fux}

hannafux@hotmail.com Cardiff University, Cardiff, Wales,

United Kingdom

\begin{abstract}
Goal: The circular economy (CE) strategy has risen as an alternative to linear systems, since it can reduce material and human footprint by introducing systemic solutions. However, there is still a huge gap of circularity, once $90 \%$ of the world resources which enter the production chain are wasted. In order to fill in this gap, it is necessary to tackle the barriers which prevent the world from implementing and improving circular initiatives. Thus, following this trend, the present work aimed to identify the key conditions for the implementation of CE initiatives, to draw an Ideal Scenario for circular economy to thrive. Design / Methodology / Approach: The present work consisted of a case study of the CircE project, hence, a qualitative research. The data collection was performed through semi-structured interviews with the representatives of this project, in addition to an analysis of the CircE project documents.

Results: Some of the results found consisted of an overview of the CircE project; key conditions for CE to thrive; and the ideal scenario for Circular Economy to thrive, draw from the analysis of these results.

Limitations of the investigation: Some limitations of the present work consisted of difficulties to access the data due to confidentiality issues of the project researched.

Practical implications: The outcome of the present work, the Ideal Scenario, represented a guideline of the steps that should be taken with stakeholders' effort, especially the government ones, to encourage the development of circular economy initiatives.

Originality / Value: The CircE project is a recent initiative in the Circular Economy field, hence very relevant to be analyzed, but with no scientific publications yet. In addition, the scientific literature regarding the implementation of $C E$ is limited, most of the existent documents concern reports of organizations. Hence, the present work aims to contribute to the scientific production on the field.
\end{abstract}

Keywords: Circular Economy; Circular Economy Gap; Barriers; Key Conditions; Ideal Scenario. 


\section{INTRODUCTION}

The current linear economic system, based on resource depletion and, on production and consumption growth, in which the materials end-up mostly on landfills and, usually follow a short life-cycle: resource - production - consumption - discard/waste; has apparently ignored the finitude of the natural stocks, as well as the consequences of such preliminaries (Jackson, 2009), namely, depletion of natural resources, environmental pollution and aggravation of the risks and effects of climate change (Wit et al., 2018). In other words, such patterns of production and consumption can no longer be sustained by the world (Berndtsson, 2015; MacArthur, 2015a).

In this sense, the circular economy (CE) strategy has risen as an alternative to linear systems (MacArthur, 2015a), since it can reduce material and human footprint by introducing systemic solutions taking into account societal needs, combining political leadership, technological innovation and behavioral changes. Thus, the economy based on circularity should consider the management of the resources, and the fulfillment of people's needs, respecting the limits of nature (Wit et al., 2018).

"(...) circular economy is an economic and industrial model that is restorative by intent and design (...) it replaces the concept of waste with the one of restoration and aims to decouple economic growth from the use of virgin resources." (MacArthur, 2015a, p. 07)

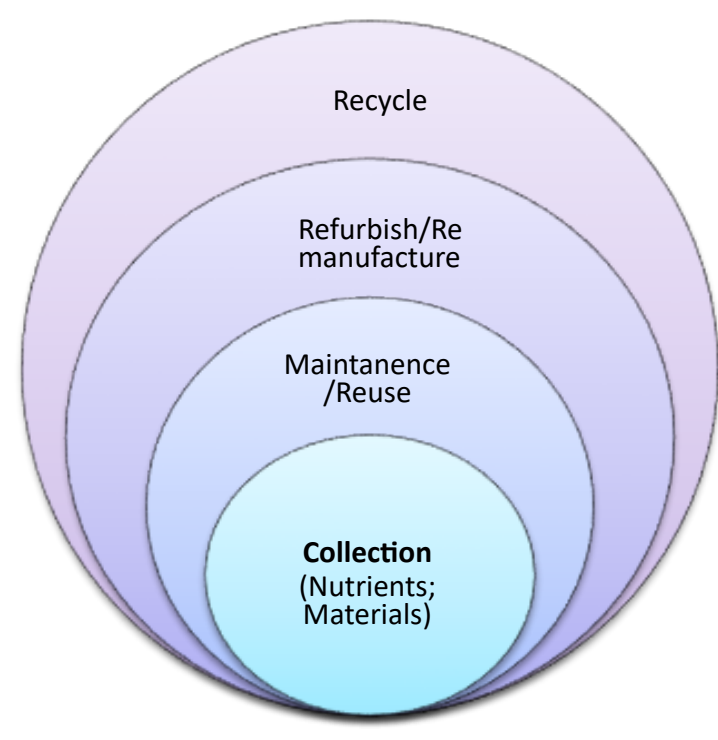

Figure 1. Strategies of circular economy. Source: author (2019), based on Macarthur, 2015a and on https://files.ontario.ca/ finalstrategywastefreeont_eng_aoda1_final-s.pdf.Accessed in March, 2018.
However, there still is a huge gap of circularity, once $90 \%$ of the resources in the world that enter the production chain are wasted, being either incinerated, landfilled or dispersed into the environment (Wit et al., 2018). Therefore, Wit et al. (2018), Macarthur (2017) and Heshmati (2015) bring up some reasons for such gap, claiming that there are several challenges for the implementation of Circular Economy, such as the insufficient support of legislations, lack of awareness about CE and its opportunities (especially because most businesses were raised in a linear economy), absence or poor cooperation along the value chain and insufficient standard systems for assessing CE's performance.

Hence, in order to fill in this gap, it is necessary to tackle the barriers which prevent the world from implementing and improving circular initiatives (MacArthur, 2017). Thus, following this trend, the present work performed a case study of the CircE project, in which key conditions for the implementation and improvement of CE initiatives were identified, aiming to draw an Ideal Scenario for circular economy to thrive.

Accordingly, the main objective of the present work consisted of: Drawing an Ideal Scenario for Circular Economy to occur, supported by the secondary Objectives:

- Recognize the criteria used to identify "Good Practices"

- Identify the key conditions for CE to thrive

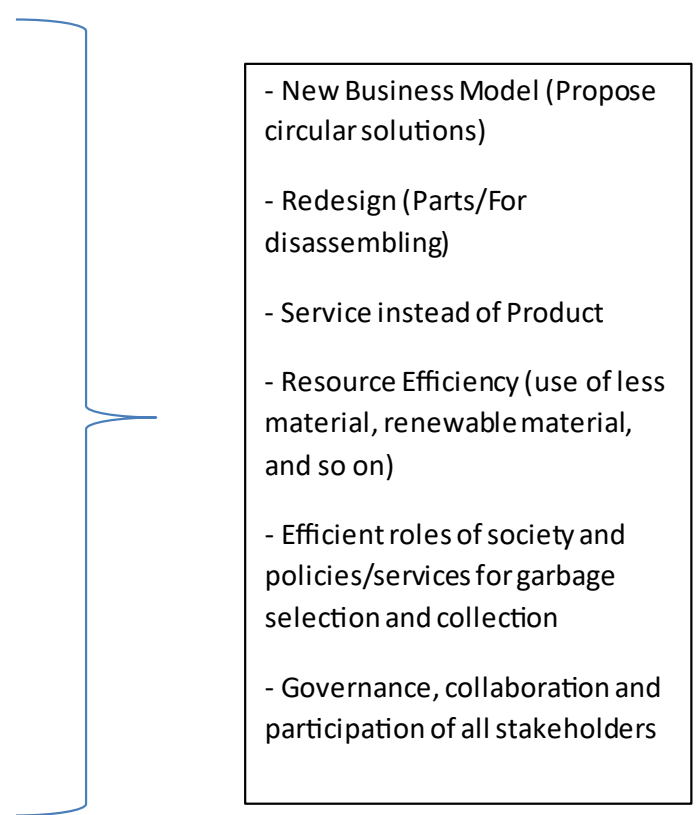


- Identify Road Blocks (Barriers)

- Analyze the existence of Action Plans (for the implementation of $\mathrm{CE}$ )

\section{METHODS}

The main focus of the present work is to research the challenges for the development of CE initiatives, from a governmental perspective and, in conclusion, draw an Ideal Scenario for its development. Therefore, the present work consists of a case study; hence, its general methodological approach is qualitative and it performed an in-depth exploration of a selected relevant case (Wisker, 2008). Thus, the CircE project was chosen to be analyzed, once it is an ongoing project that tackles these challenges from the perspective of eight EU countries, aiming to strengthen Circular Economy (CE) in Europe, through the exchange of knowledge and experience among the partners, thereby improving the range of their policy instruments to steer the economy towards the CE model, also stimulating the involvement of stakeholders in CE initiatives.

Moreover, once the CircE project is composed by 8 partners of 8 European countries: Lombardy Region, Government of Catalonia, Marshal's Office of Lower Silesia, Province of Gelderland, London Waste and Recycling Board (LWARB), Creation Development EcoEntrerprises (CD2E), Sofia Municipality, Association of Municipalities and Towns of Slovenia (SOS); it provided a good sample for circular economy analysis. The collection of data was performed through semi-structured interviews, aiming to collect primary data from representatives of these 8 partners. However, it was possible to conduct the interviews with the representatives of only 5 of the 8 partner members of the CircE project, to gather in-depth information over Circular Economy. Finally, in addition to that, secondary data was also assessed through the analysis of the documents and reports regarding the 8 partner members of the CircE project.

The main research question of the present work is: "What is the ideal scenario for circular economy to occur, according to the CircE project stakeholders?" This question was supported by 4 sub-questions. The following table shows the separation of the methods that were used to answer the 4 sub-questions, which supported the response of the main research question.
Table 01. Research methods according to the research questions. Source: created by the author.

\begin{tabular}{|c|c|c|}
\hline RESEARCH QUESTIONS & $\begin{array}{c}\text { REPORTS AND } \\
\text { DOCUMENT } \\
\text { ANALYSIS }\end{array}$ & $\begin{array}{c}\text { SEMI-STRUC- } \\
\text { TURED INTER- } \\
\text { VIEWS }\end{array}$ \\
\hline $\begin{array}{c}1-\text { What are the criteria used } \\
\text { to identify "Good practices" } \\
\text { of Circular Economy? }\end{array}$ & $\mathrm{x}$ & $\mathrm{x}$ \\
\hline $\begin{array}{c}\text { 2- What are the Key Con- } \\
\text { ditions identified for CE to } \\
\text { thrive? }\end{array}$ & $\mathrm{x}$ & $\mathrm{x}$ \\
\hline $\begin{array}{c}3 \text { - What are the Road Blocks } \\
\text { identified? }\end{array}$ & $\mathrm{x}$ & $\mathrm{x}$ \\
\hline $\begin{array}{c}4 \text { - What is the Action Plan } \\
\text { used for the implementation } \\
\text { of CE? }\end{array}$ & $-\ldots$ & \\
\hline
\end{tabular}

\section{RESULTS}

\section{Introduction}

Considering Circular Economy as a viable alternative to implement more sustainable patterns of production and consumption, the present work, which has a more government-oriented approach, aimed to verify the barriers preventing the circularity gap to be bridged and also, to propose possible solutions, to finally draw an ideal scenario for circular economy to develop. Therefore, the common grounds and strategies for the implementation of circular economy among the partners within the CircE project were identified.

Moreover, for analysis purpose, the CircE project partners researched were classified according to the country they represent, namely, UK partner, Italy partner, Netherlands partner, Poland partner, Bulgaria partner, Spain partner, France partner, and Slovenia partner.

In this sense, a general overview of the project with an in-depth analysis was performed, in order to answer the central question identified in the present work, "What is the ideal scenario for Circular Economy to occur, according to CircE Project stakeholders", and its 4 sub-questions mentioned above. Hence, the findings were organized based on the research questions and outcomes:

- Overview of the CircE project;

- Focus Sector;

- Criteria used to identify "Good Practices";

- Road Blocks (Barriers); 
- key conditions for CE to thrive;

- Action Plans (for the implementation of CE);

- The IDEAL SCENARIO for Circular Economy to thrive.

\section{Overview of the CircE project}

CircE is an ongoing project that started in 2017 and will finish in 2021; hence, there are some steps that were already performed and others that are still in the process of development. Thus, the current stage, mapping, is essential for the project, and aims to cover aspects such as, the identification of actors (stakeholders), good Practices, barriers, opportunities, and so on. These aspects are important to increase the knowledge of the contexts regarding circular economy and also the comprehension of how ready the region is to move forward in circular economy, as well as what still needs to be addressed.

Thereon, after the mapping stage, in which the opportunities and barriers of circular economy were identified, every partner will create an action plan as a final outcome of the project to tackle these issues, according to their particular contexts. Besides that, this information will support the development or improvement of policies regarding circular economy. Finally, the action plan, together with the policy support will provide suitable instruments for circular economy to thrive in their regions.

\section{Focus Sector}

The division of circular economy initiatives in sectors is a common strategy to simplify and optimize the work with $\mathrm{CE}$, especially from a macro perspective, such as, the government one. Thus, every sector of CE initiatives has specific features and requires a set of actions to address them. Hence, the CircE project selected sectors of interest that are relevant for all the regions involved in the project regarding circular economy, which consisted of the focus sectors: built environment; plastics; food waste; textile; WEEE-strategic metals; tourism; biomass; raw materials. These sectors were chosen based on criteria of economic interest and potential opportunities to develop circular economy in all the project partners. Afterwards, the partners gathered in groups, according to the sectors selected, to share specific knowledge, difficulties, and experiences from their focus sectors. Nevertheless, it is important to note that each partner is researching their area of interest, choosing about 3 sectors (not all the 8 sectors), which are more relevant for their region.-Therefore, among all the eight CircE partners, the built environment was the most common sector, being chosen by most of them - 5 of the partners, in addition to 1 partner that identified the raw material as a sector, but which is also related to the built environment. The second most identified sector was textile ( 5 partners), followed by food waste, plastics, and tourism. The remaining sectors, WEEE-strategic metals; biomass; raw materials appeared less frequently among the CircE partners. For instance, the Netherland partner focused on textile, built Environment, and biomass; the Italy partner focused on built environment, biomass, and food waste, and so on.

Therefore, in order to identify and select such sectors the partners engaged in some processes for data collection; in other words, the selection of the source from which the data was collected was based on few criteria. Hence, the CircE project identified that circular economy is frequently connected with innovation, and also waste management, job, and income generation, etc. Thus, the project collected information regarding the Smart Specialization Strategy (analysis of the potentialities of the places, especially the technological ones, together with an approach for their implementation), reports and initiatives. Besides that, several other sources were contacted, such as, environmental organs, statistical institutes, water and waste management organizations, in order to collect data regarding the chosen sectors in each region, namely, number of employees and companies in the region, Gross Value Added by the activity, resource productivity, volume of waste generated, amount of waste reused/remanufactured, recycled, incinerated, landfilled, energy consumption, and so on.

In this sense, it can be said that the criteria used by the CircE project to identify circular economy initiatives, hence, the focus sectors, consisted of innovation, economic relevance and waste generation aspects. Thus, the sectors that presented more relevant numbers related to such criteria were selected accordingly in each region.

\section{Criteria used to identify "Good Practices"}

The concept of good practices created by the CircE project is central for the project. Thus, good practices consists of circular economy initiatives identified by every one of the regions, regarding the focus sectors selected by each partner, concerning educational (universities, organizations and initiatives that promote $\mathrm{CE}$ ), policies (related to waste management or CE-oriented) and, technical aspects (for instance, innovation, use of waste as resource, services instead of products, and so on).

Moreover, the report Toolkit for Policymakers (Macarthur, 2015b), which provided a framework of technical aspects of circular economy, has supported the methodology of the CircE project and guided the identification of good practices. Hence, such technical aspects, based on the re-evaluation 
of the resource use and the product concept, consisted of: regeneration (use of renewable materials and energy), sharing (keeping the product loop - maximizing its utilization, sharing with different users), optimization (increasing performance and efficiency of a product and process of manufacturing), looping (keeping components and materials in a closed loop), virtualization (dematerialization), exchange (new products, services and technologies, renewable materials) (Macarthur, 2015b). Therefore, the initiatives that performed one or some of these behaviors could be mapped as good practice of circular economy. Such features, commonly found in innovative practices, together with aspects related with new business models (oriented to circularity or sustainability), as well as educational, policies, technological initiatives, and information regarding waste (generation, recycling, and so on) were also used as indicators of CE. Besides that, as previously mentioned, the CircE project recognizes that very often CE is related to innovation; hence, Smart Specialization initiatives also helped identify good practices.

However, although the criteria for the identification of good practices was the same for all the partners, the recognition of such initiatives did not happen equally in all the partner members of the project. Each partner belongs to a different region and, thus, is inserted in a different context, having different features, availability of data, and also diverse levels of understanding of the concept of circular economy. In this sense, some regions have more awareness of the circular economy concept; therefore, there are more initiatives to be mapped, while others faced more difficulty to identify such initiatives due to the lack of information regarding CE. Thus, the mapping of good practices was smoother and more straightforward in the regions where the awareness of CE is higher, namely, Spain Partner, Italy Partner, and the Netherlands Partner. Therefore, the identification of Good Practices was performed through benchmarking, searching for initiatives on the internet, stablishing direct contact with them or through the umbrella organization, clusters, educational institutions or representative organizations of the sectors, for instance. On the other hand, in the partner regions where the CE awareness is lower, the process of identification of good practices faced more challenges, namely Poland Partner and Bulgaria Partner. Thus, these last partners had to dig a little deeper to find the CE initiatives, using strategies, such as researching Smart Specialization reports and initiatives, contacting several sources of data to find indicators of $\mathrm{CE}$, as, for example, environmental organs, statistical institutes, and water and waste management organizations.

The mapping of good practices was carried out based on the focus sectors of each region, which throws light on the currently situation of the circular economy in the region, the achievements reached so far and the impediments for the development of circular economy.

\section{Road Blocks (Barriers)}

Furthermore, although the CircE project separates opportunities and barriers, their core is the same, once opportunities arise where barriers can be overcome. Hence, opportunities, good practices and barriers are strongly related in this project; thus, while identifying the good existent practices, the CircE partners recognized where there was room for improvement, regarding the development of circular economy. Afterwards, while mapping opportunities, the barriers to achieve them were also recognized.

Therefore, some of the opportunities/barriers are: business can be reluctant to changes, fearing possible losses, especially regarding the necessary investments for the circular transition (Poland Partner). In addition to that, the high prices of some circular resources, such as recycled textile and insufficient technology, in comparison with linear (raw) resources, are also the road blocks encountered (Netherland Partner).

\begin{abstract}
“(...) consumers are not ready for recycled material products, it is too expensive to recycle, and they rather buy cheaper products. It is not easy to change consumer behavior. A solution for textile could be refunding money when products are brought back. It is also necessary to promote campaigns, so recycled clothes become more popular. Besides that, it is important to reduce prices of recycling textile - in other words, more breakthrough technologies are necessary." (Netherlands partner, 2018)
\end{abstract}

Moreover, most partners agree that business and consumer awareness can be a substantial road block to the development of $\mathrm{CE}$; hence, behavior change towards circularity represents a challenge to be tackled. Accordingly, the Spain partner and the Polish partner presented some examples of events (seminars, meetings) that are being promoted with this purpose, to deal with the lack of awareness and fear of failure of the business initiatives in the region. For instance, in these events, successful CE businesses present their positive results, helping to expand CE knowledge and also stimulating cooperation and networks among stakeholders.

In this sense, copping with the opportunities/barriers mapped up to now will require a lot of investment, especially, in financial and knowledge terms, demanding the involvement of several stakeholders, for instance, governments (through action plans, policies, tax systems), business (cooperation, changing behavior, and other technical aspects) and consumers (shifting consumption and discard patterns). 
Brazilian Journal of Operations \& Production Management

Volume 16, Número 1, 2019, pp. 157-165

DOI: 10.14488/BJOPM.2019.v16.n1.a15

\section{Key conditions for CE to thrive}

The concept of key conditions was created within the present work, based on the data collected in the research of the CircE project, and it is related to the information concerning the good practices, opportunities and barriers, which are, as mentioned before, strongly connected, being able to provide valuable information about each other. Hence, the key conditions consisted of a reunion of the most relevant and common aspects identified among the CircE partners to be tackled by the governments of the CircE partners in cooperation with the stakeholders, especially the business ones to promote the development of circular economy in their regions, respectively.

Therefore, the key conditions recognized were: good consumers and business awareness and behaviors oriented towards $C E$, such as, promotion of CE events involving several stakeholders; waste management policies and strategies, in order to make feasible the availability of secondary resources; policies to reinforce CE aspects (for instance, tax reduction for CE businesses); training and educational regarding circular economy, in order to both qualify human resources to tackle the new demands and, to develop scientific research in the field; good communication, cooperation and networks among stakeholders (information sharing, CE awareness, disclosure of CE business, demand/offer systems, and so on); investment in new technology and innovation (new products, business, more efficient use of resources, recycling); and also specific metrics to measure circular economy progress (although CircE recognizes these metrics as essential, the project does not aim to generate such measures).

For instance, the Spain partner named some actions that are being implemented by the CircE partners, and specifically in its region, in order to reach the key conditions for the development of CE.

\begin{abstract}
"Meetings with the stakeholders; posting reports and other information in the CircE website; establishing collaborations and network for CE; training and increasing CE awareness, collaborating with specific public authorities; Market Place (events where businesses can show themselves and, people can go to look for products and services); and organizing events to increase awareness and share knowledge, such as, Mapping Week (international initiative to map CE initiatives)." (Spain partner, 2018)
\end{abstract}

Moreover, the promotion of meetings was identified by the partners as a very important action, and as part of the key conditions, to improve awareness among stakeholders, and encourage partnerships and change of behavior.

\begin{abstract}
"For example, we are doing a conference on the $21^{\text {st }}$ of May evolving the circular economy in our region. We tried to show examples of some companies that are using the concept of circular economy, and it is working, they are making money with it." (Poland partner, 2018)
\end{abstract}

Finally, the key conditions include the most important aspects for the development of $\mathrm{CE}$, which, in short, aim to: improve the awareness of $C E$, the availability of secondary material (waste as resource), involving universities and other educational institutions to capacitate manpower to CE demands, as well as developing sustainable innovation and technology, besides promoting cooperation and networks among the stakeholders, through the development of certain actions, such as, events, projects and platforms. In other words, the key conditions serve as a guideline for governmental organizations to improve and implement CE initiatives, containing the necessary actions to be taken, accordingly and, forming an Ideal Scenario for CE to move forward.

\section{Action Plans (for the implementation of CE)}

The CircE project partners, in their mapping stage described above identified essential actions that have to be implemented in order to achieve successful development of circular economy. Hence, all the project partners interviewed recognized the need of an action plan for circular economy. However, from the five project partners interviewed (Spain, Netherlands, Poland, Italy and Bulgaria), only the Netherlands partner claimed the existence of a specific action plan for circular economy, which, in its perception, still needs to be improved.

\begin{abstract}
"Although we have an action plan, there is still need to improve the existent policies and public initiatives, to increase the amount of waste recycled and its use in building materials, for instance. The implementation of circular economy is a long term process and the creation of small and big projects and initiatives are necessary to scale up the development of CE in the local, regional and national levels." (Netherlands partner, 2018)
\end{abstract}

Thus, the mapping of good practices, opportunities and barriers by the CircE project will provide the essential information to guide the design of the action plans in the regions. In other words, the action plan will gather all the information regarding the circular economy identified by the project, providing strategies which must be implemented accordingly by each sector, aiming at increasing the amount CE initiatives, the improvement of the existent ones and also the development of circular economy in general, in accordance with each region context. 
Therefore, the desired outcomes of the CircE project, necessary for CE to thrive, consists of the development of action plans, unifying the strategies, in coherence with each place where CE is implemented, besides the creation and advance of policies to support and reinforce the actions to put CE forward and change business and consumer behaviors. Accordingly, CircE project aims to bridge the existent gap of circularity by implementing a set of strategies, identify as key conditions for the success of CE.

\section{The IDEAL SCENARIO for Circular Economy to thrive}

In order to bridge the gap of circularity, the governments play an essential role that ranges from mapping good practices (existent circular economy initiatives) to identify key conditions for CE to occur, as well as the road blocks for the implementation of the CE initiatives (Wit et al., 2018; Macarthur, 2017). Thus, the identification of such aspects and the implementation of strategies to tackle them require the accomplishment of several steps, which may or may not follow an order, that were congruently recognized by Wit et al. (2018) and Macarthur, (2017) and also in the CircE project.

In this sense, the key conditions, which are steps recognized by the present work that are necessary to be taken for CE to occur, and which are based on the opportunities and barriers commonly identified within the CircE project, consist of:

- Waste management (it increases public awareness for the separation of waste and develop policies and strategies for its collection and later availability as resource, for instance);

- Use of waste as resource (it changes the perspective over waste and its destination, for instance, re-using, recycling, introduction of percentage of secondary raw material in products);

- Improve CE awareness from business and consumers (through campaigns, meetings, seminars, education, and courses, for instance, it spreads the concept of CE, as well as, the opportunities, strategies, and possibilities);

- Training and Education (implementation of courses and development of knowledge, varying from high education and scientific research to training for the development of CE skills);

- Creation of metrics to assess CE performance and progress;
- Development of platforms to promote communication among stakeholders (it promotes knowledge exchange and access to data concerning waste/resource procurement, offer and demand);

- Stimulation of the development of cooperation and networks among stakeholders (businesses, governmental organizations, and consumers);

- Policies, action plans and guidelines to guide the implementation of CE initiatives, to promote changes of both business and consumer behaviors into $C E$ direction as well (For example: tax reduction for $\mathrm{CE}$ companies);

- Development of CE oriented industries, entrepreneurships, start-ups (CE initiatives new business models, services, products and, improvement of technology and innovation);

- Local Production (in order to reduce human footprint, avoid importation, stimulating and prioritizing local initiatives);

- Resource efficiency and use of cleaner and greener resources (It promotes better use of the resources, through zero emission, composting, waste as resource, electric vehicles, biomass, bioproducts, natural fibres, and so on).

Moreover, it is important to state that these key conditions can be implemented simultaneously or even overlap, meaning that there is no specific order for their development (Fux, 2018). Furthermore, the success of circular economy through the completion of the key conditions, demands the cooperation and gathered efforts of their stakeholders, especially of the policymakers at all levels (city, national and regional). Hence, in order to improve the previously mentioned areas, allowing CE to thrive, it is necessary the existence of government strategies to support business initiatives, educational organizations, innovations and technologies, as well as, to steer business and consumer behaviors towards circularity (Fux, 2018; Wit et al., 2018; Macarthur, 2017).

Therefore, after the analysis of the data collected from the CircE project, an ideal scenario for circular economy to thrive could be drawn more thoroughly, since this data provided more detached information over circular economy, from the perspective of government stakeholders who are dealing directly with CE mapping and the implementation of solutions, accordingly. Thus, this ideal scenario should contain the key conditions that are necessary to be tackled in order to develop CE in those regions, as mentioned above. Finally, see below the "Ideal Scenario"- the research outcome of the present work: 


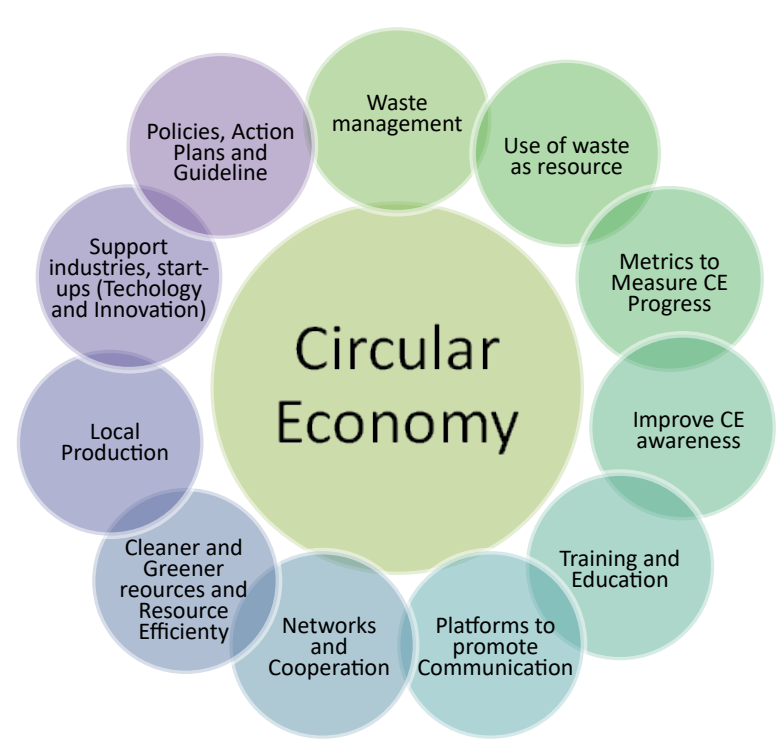

Figure 2. Ideal Scenario: Research Outcome. Source: Fux, 2018.

\section{CONCLUSION}

Circular economy consists of a sustainable production and consumption model that is considered a viable alternative for the current linear one and its dreadful consequences for human life and the environment. In other words, the circular economy model offers economic opportunities, creating new jobs and businesses, besides providing the necessary means for human survival, and mitigating climate change effects, through the reduction of waste and more efficient use of resources.

Despite the innumerous benefits of implementing circular economy, it is not yet widely spread around the world. Several circular economy reports, such as the Circular Economy Gap Report, claim that there is a 90\% gap in the circularity of resources; in other words, only about $10 \%$ of the resources that enter the production system come back to it - in a circular way - for example, through reverse logistics, recycling, re-using, and so on. Therefore, in order to achieve higher levels of circularity, more effective solutions should be put in practice, including the creation of better policies concerning $\mathrm{CE}$ and the reinforcement of the existing ones, and addition to the development of action plans for its implementation.

Regardless of the existence of this huge gap circularity and the awareness that a lot is yet to be done, there are many projects related to circular economy taking place all over the world, especially in Europe, where countries such as Netherlands, Italy, UK, and others that are considered the main reference in the field, are located.
Thus, following this trend, the present work performed a case study of the CircE project, a circular economy project of the European Union initiative. This case consisted of a valuable source of data regarding the circular economy over its good practices, opportunities and barriers, addressing the development of more effective policies and action plans, aiming to increase the amount of CE initiatives and to improve the necessary conditions for it to flourish. Hence, the present work answered the four research questions proposed initially:

1. What are the criteria used to identify the "Good practices" of Circular Economy?

2. What are the Key Conditions identified for CE to thrive?

3. What are the Road Blocks identified?

4. What is the Action Plan used for the implementation of $\mathrm{CE}$ ?

Moreover, the knowledge acquired in the present work, regarding the CircE project, provided the necessary information, containing key conditions for the CE success, to draw an ideal scenario for CE to thrive.

Accordingly, the Ideal Scenario designed relies on key conditions that consist of a set of overlapping steps that should be taken (ideally) simultaneously, counting on the cooperation and gathered efforts of the stakeholders, especially business initiatives and policymakers. Hence, these steps include the improvement of waste management policies and strategies; the use of waste as resource; increase of CE awareness from business and consumers; training and education; development of metrics to measure CE performance and progress (it was not the focus of CircE project, although the project recognized its importance); creation of platforms to promote communication among businesses and also consumers; stimulation of networks and cooperation among stakeholders - businesses, consumers and governmental organizations; policies, action plans and guidelines to steer both consumers' and businesses' behaviors into CE direction; support industries, entrepreneurships, start-ups; local Production; cleaner and greener resources and resource efficiency. In short, the implementation of the key conditions contained in the Ideal Scenario provides macro strategies to be supported by the stakeholders, especially by the government strategies, which will allow the development of actions to promote circular economy, enabling the increase of initiatives where the ideal scenario is developed, taking into account the features of the context. 


\section{LIMITATIONS}

The present work aimed to create a practical and useful guide for the implementation of aspects to support circular economy, allowing it to move forward, especially from the perspective of governments. Therefore, the analysis of the CircE project provided valuable data to do so. Despite the efforts, as seen before, it was not possible to collect all the desired information. Besides that, once the CircE project desired outcomes are the development of better policies (CE oriented) and CE action plans, it would be resourceful to look deeper into the existent policies related to the topic, and also check the existent action plans of the regions researched, in order to compare the mapped opportunities with the gaps in these documents. Although most of the CircE partner regions do not contain specific action plans for $\mathrm{CE}$, aiming to create them by the end of the project, the assessment of the policies would provide very useful data. Thus, the analysis of such documents, for instance, policies regarding waste management, circular economy and any other related fields, such as sustainable strategies and smart specialization, could provide valuable insights over circular economy and the existent restraints. However, due to time constraints and the volume of documents that should be assessed, it was not possible to make an in-depth review of them, since the amount work required by such analysis would not be consistent with the schedule of the present research.

\section{REFERENCES}

Berndtsson, M. (2015), Circular Economy and Sustainable Development (Master's thesis).

Fux, H. (2018). What is the ideal scenario for circular economy to occur? A case study of the circe project (Master's thesis).
Heshmati, A. (2015), A Review of the Circular Economy and its Implementation. International Journal of Green Economics, Vol. 11, No. 6911.

Jackson, T. (2009). Prosperity without growth: Economics for a finite planet. Routledge.

MacArthur, E. (2015a). Circularity indicators: An Approach to Measuring Circularity. Methodology. Publication of Ellen Macarthur Foundation.

MacArthur, E. (2015b). Delivering the Circular Economy - A Toolkit for Policymakers. Publication of Ellen Macarthur Foundation.

MacArthur, E. (2017). Achieving "Growth Within". Publication of Ellen Macarthur Foundation and Sun Institute Environment and Sustainbility.

Wisker, G. (2008). The postgraduate research handbook: Succeed with your MA, MPhil, EdD and PhD. Palgrave Macmillan.

Wit, M., Hoogzaad, J., Ramkumar, S., Friedl, H., Douma, A. (2018). The Circularity Gap Report: An analysis of the circular state of the global economy. Publication of Circle Economy.

\section{AKNOWLEDGMENT}

This article is based on the results achieved in the dissertation "What is the Ideal Scenario for Circular Economy to Occur? A Case Study of the CircE Project" of the Planet Europe master's program (June, 2018). Hence, it contains some of the data collected within this work, and it may have similar perspectives. However, the article provides a unique point of view built for it. Moreover, the information regarding the CircE project are referent to the case study performed within the dissertation; thus, it may contain different aspects from the ones proposed by the CircE project per se.

Received: 05 Oct 2018

Approved: 15 Jan 2019

DOI: 10.14488/BJOPM.2019.v16.n1.a15

How to cite: Fux, Hanna (2019), "What is the ideal scenario for circular economy to occur? A case study of the circe project", Brazilian Journal of Operations \& Production Management, Vol. 16, No. 1, pp. 157-165, available from: https://bjopm.emnuvens.com.br/bjopm/article/view/745 (access year month day). 